\title{
Control strategy for networked control systems with time delay and packet dropout using linear matrix inequalities
}

\author{
Zhaohong Wang ${ }^{*}$ and Seiji Fukushima
}

\begin{abstract}
A control strategy using linear matrix inequalities towards time delay and stochastic packet dropout of networked control system is proposed in this paper. First, a reconfigured target plant of networked control system is built based on a linear time-invariant target. Before discretization of target plant, a Pade approximation is employed towards the time delay segment of reconfigured target plant, while zero-order holder compensator and an estimator are employed for packet dropout of control input and measured output, respectively. Then the control target is reformed as a programming problem of an uncertain time-variant multi-parametric state space. In order to deal with such problem, herein the time-variant state space is rewritten as a linear parametric-varying prediction model by enumerating all possible occasions. Afterwards, a novel robust model predictive controller is proposed as a constrained minimax optimization of the predictive cost function. Finally, we present two simulations based on an aircraft model and a second-order numeric model, by which step responses and explicit solutions are derived, performed and analysed. Simulation results demonstrate both the stability and robustness of the proposed control strategy.
\end{abstract}

Keywords: Networked control system, Time delay, Packet dropout, Model predictive control

\section{Introduction}

Alongside of the development of Internet of Things (IoT), we have paid more and more attention to networked control systems (NCSs), wherein the closed control loops are connected via networks [1]. Meanwhile, enhancement of system on chip $(\mathrm{SoC})$ and cloud computing have stimulated concerning over practical low-power networks, requiring for both security and low-power consumption, for instance, Bluetooth Low Energy (BLE), Zigbee, etc. [2]. Nowadays, NCSs are becoming more and more popular in both industry and daily life. Nevertheless, we have to beware of two main defects that networked control systems may have: problems such as time delay and packet dropout. Considering time delay and packet dropout in NCSs, the reasons for them are varied, some are due to distance, and others are due to disturbances or intrinsic property. For example, Bluetooth has an intrinsic time delay even if a face-to-face connection. Fortunately, time delay and packet dropout can be

\footnotetext{
* Correspondence: jvkhong@sina.com

Graduate School of Science and Engineering, Kagoshima University, Kagoshima, Japan
}

ignored in most cases, and some of the rest may be compensated by a more robust connection. However, in the NCS requiring for higher real-time characteristics, time delay and packet dropout should be taken into consideration of control strategy.

Research on NCSs is generally categorized into two aspects: control of network and control over network. The first aspect includes measures making networks suitable for real-time NCSs, e.g. congestion reduction and efficient networking protocol. Meantime, control over network pays more attention to control strategies and control systems design over the network to enhance the performance of control system against packet dropout, time delay, etc. [3]. Our study is under the domain of control over network. While in this area, there have been many remarkable studies discussing such issue of time delay and packet dropout in NCSs. Mehrivash and Shafiei have discussed a robust model predictive control for discrete-time delayed systems, when most practical networked control systems are continuoustime delayed [4]. Torres et al. have considered a networked control system with time delayed less than sampling period, 
the application area of which is limited and the study is more concerning over modelling [5]. An explanation of Markov chains process in packet dropout has been introduced by [6]. Seiler and Sengupta modelled packet dropout as a Markov jumping linear system and discussed an $\mathrm{H}-\infty$ approach for it. The global $\mathrm{H}-\infty$ gain is excellent; however, an approximation of bilinear matrix inequality problem is not well proved [7]. A standard model predictive controller has been raised with fast response speed in 40 steps but the robustness of such control design has not been discussed [8]. Nesic and Teel have employed a perturbation theory to analyse the stability of NCSs that could be applied in the forward path from a sensor to a controller, although it is not feasible to approximate the system as a continuous one in a real control practice [9]. Yang and Han discussed an $\mathrm{H}-\infty$ controller solved by linear matrix inequalities at a significantly low cost, while the entire control system is at a risk of being instable since only optimal situations are under consideration, leaving alone the worst situations [10]. A modified preview control for a wireless tracking control system with packet loss is proposed by [11] in need of some deterministic future segment while most NCSs in reality have only stochastic future.

As is shown in Fig. 1, the overall NCS consisted of target plant, controller and network. Afterwards, the NCS is reconstructed by merging target plant and network into a reconfigured target plant in Fig. 2. In the modelling process, segments of time delay are eliminated through Pade approximation. Then a zero-order holder compensator is proposed for packet dropout in control input, while packet dropout in measured output is compensated by estimation. Then the control target is reformed as a control problem of an uncertain time-varying multi-parametric state space. Moreover, in order to apply toolbox YALMIP and solver MPT3, such state space is rewritten as a linear parametric-varying prediction model. Finally, a robust model predictive controller is completed by solving a constrained minimax problem of the predictive cost function.

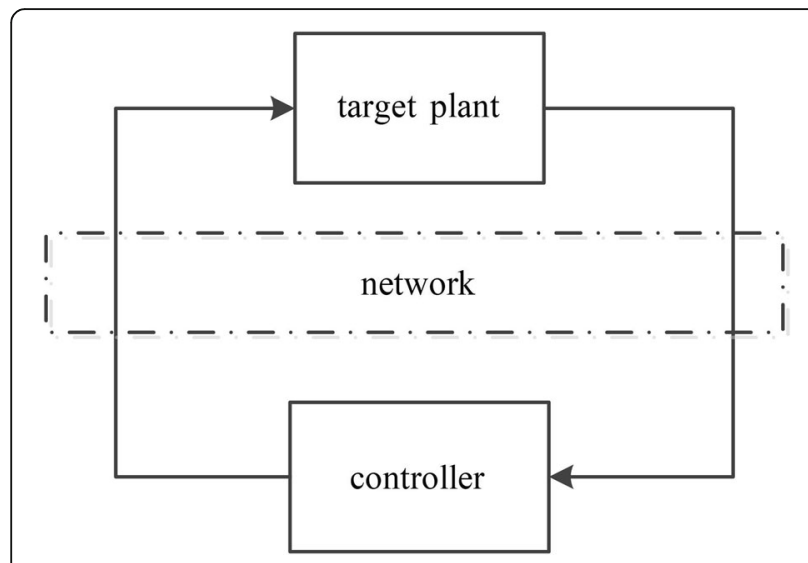

Fig. 1 Brief diagram of a single-loop networked control system

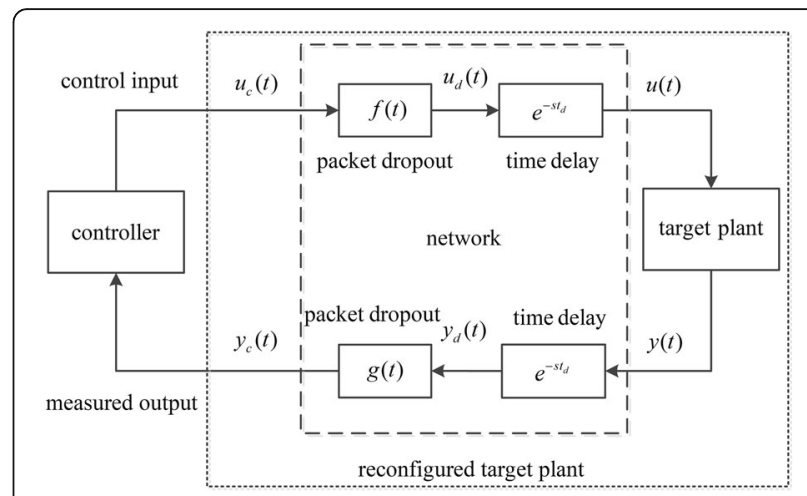

Fig. 2 Reconfiguration of networked control system

Two numeric simulations in Simulink are presented in Section 5.

Model predictive control is widely used both in theory and in practice [12]. The main shortcoming of MPC is the computational expense required to solve the constrained finite-time optimal control (CFTOC) problem, which prevents the application of MPC with a high sampling rate and is expensive to be achieve, since the necessary computational equipment and higher power consumption has to be provided, which is critical in NCSs, especially in wireless ones. In a view of past research and existing literature, our study highlights in the following:

1. Packet dropout is modelled as binomial distribution and a zero-order holder compensator is designed for better performance.

2. The worst occasions of future states are taken into consideration via a minimax optimization problem of a model predictive controller, by which the robustness of the proposed method is promoted.

3. Computational expense has been reduced adequately since a discrete dynamic programming is designed in solution codes.

The structure of this paper is organized as follows. Section 2 shows the control problem formatted from typical NCSs. In Section 3, modelling of both packet dropout and time delay in NCSs is discussed alongside with a compensator proposed for the packet dropout. Section 4 discusses the detailed design of robust model predictive controller. Two simulations are presented in Section 5 as well as the discussion and conclusion of them.

\section{Problem formation}

Whatever the structure and complexity of NCSs could be, research on such NCSs consisted of numerous sensors, actuators and controllers begins with every single-loop NCS shown in Fig. 1. Not like conventional unnetworked 
control systems, NCSs usually suffer from packet dropout and/or time delay. These abnormalities may incur instability or latency to the control system. In large dimensional NCSs, here, we discuss control strategies with respects to both packet dropout and time delay in networked control system. For convenience, NCS in this study is referred to the single-loop NCS shown in Fig. 1.

A detailed NCS is shown in Fig. 2, in which both data transmissions of control input and measured output are networked, both suffer from packet dropout and time delay. Since the issue we focus on is the network segment, it is decomposed into four segments of packet dropout and time delay as is shown in Fig. 2. Furthermore, segments of the target plant and network are merged and reconfigured into a new target plant. Meanwhile, a linear time-invariant (LTI) target plant is considered with whose state space is

$$
\left\{\begin{array}{l}
x(t)=A x(t)+B u(t) \\
y(t)=C x(t)
\end{array}\right.
$$

where $x(t), u(t)$ and $y(t)$ are states, input and output of the target plant, respectively, while $A, B$ and $C$ are transition matrix, input gain and output gain of the LTI system.

As shown in the network segment of Fig. 2, the sequential order of time delay and packet dropout may be ignored due to the commutative property of multiplication. Therefore, the positions of time delay and packet dropout can be switched. In order to keep the consistency, two time delay segments are placed coherently to the target plant, while packet dropout segments coherently to the controller. The time delay equations are

$$
\left\{\begin{array}{l}
u(t)=u_{d}\left(t-t_{d}\right) \\
y_{d}(t)=y\left(t-t_{d}\right)
\end{array}\right.
$$

where $t_{d}$ is the delayed time in NCS. Not like the packet dropout, the delayed time in NCSs is not affected by circumstance a lot, so it is usually assumed to be a constant known to us in a lot of previous research [13]. $u_{d}(t)$ is a variable designated for control input before time delay, while $y_{d}(t)$ is a variable designated for measured output after time delay.

Correspondingly, the dropout equations of packet dropout are modelled as

$$
\left\{\begin{array}{l}
u_{d}(t)=f(t) u_{c}(t) \\
y_{c}(t)=g(t) y_{d}(t)
\end{array}\right.
$$

where $f(t)$ and $g(t)$ are uncertain binary dropout functions irrelevant to each other and $u_{c}(t)$ is the control input derived directly from controller, while $y_{c}(t)$ is the very measured output controller receives. Packet dropout out takes place when the values of them are zero, vice versa. In a view of practical instance of networked control system, data transmission is usually conveyed in packets intermittently at every transmission interval. As a result, dropout functions are actually discrete functions of massive dots. For convenience, $f(t)$ and $g(t)$ are usually proposed as a piecewise-defined function. Though the overall networked control system in Fig. 2 differs from a normal control system consisting of only target plant and controller, we may reconfigure it with the help of Eqs. (1)-(3) by merging segments of the target plant and network into a new reconfigured target plant, the state space of which is derived as

$$
\left\{\begin{array}{c}
x(t)=A x(t)+f\left(t-t_{d}\right) B u_{c}\left(t-t_{d}\right) \\
y_{c}(t)=g(t) C x\left(t-t_{d}\right)
\end{array}\right.
$$

The control problem of NCS is consequently reformed as a control problem of a non-linear delayed target plant of state space (4). The control target of our study is to model and stabilize the reconfigured target plant (4), seeking an enhanced performance in such NCSs.

\section{Method}

Since the control problem is formatted in Section 2, a further modelling process is required, not only for the prediction model of model predictive controller but also a prerequisite for the usage of YALMIP toolbox in the fourth chapter. There are three steps in this modelling process. Discretization is the very first. The reconfigured target plant is actually a hybrid system of both continuous and discrete, when studying the dynamics of reconfigured networked control system in (4). Whereas in typical networked control systems, data is transmitted in packets every transmission interval, so it is rational to discretize the networked control system at the transmission period, designated as $T_{s}, T_{s}<t_{d}$. However, the time delay segments, of which the delayed time be utilized as sampling rate in normal occasion, is not easy to handle in such dual-time-rate system of $T_{s}$ and $t_{d}$. Therefore, secondly, an augmented state space by Pade approximation is employed to eliminate the time delay. Furthermore, a compensator of zero-order holder is designed for the packet dropout in control input, as well as the estimation for the measured output proposed at the last.

For the time delay, Pade approximation is utilized for modelling. Among a few approximation methods, Pade approximation may still work when the target function's Taylor series does not converge, and it is widely used in computer calculation especially in rational functions of the given order [14]. Since parameters $A$ and $B$ in (1) are constantly known to us, discretized transition function of orthe iginal target plan and time delay in control input at sampling rate $T_{s}$ is supposed to be 


$$
x(k+1)=A_{d} x(k)+B_{d} U_{d}(k)
$$

where $A_{d}$ and $B_{d}$ are corresponding discrete parameters, mark $U_{d}(t)=u_{d}\left(t-t_{d}\right)$. Then a discrete Pade approximation is applied to $U_{d}(k)$ as

$$
\left\{\begin{array}{l}
U_{d}(k)=C_{\phi} \phi(k)+D_{\phi} u_{d}(k) \\
\phi(k+1)=A_{\phi d} \phi(k)+B_{\phi d} u_{d}(k)
\end{array}\right.
$$

Similarly, a Pade approximation in measured output can be written as

$$
\left\{\begin{array}{l}
X(k)=C_{\phi} \phi(k)+D_{\phi} x(k) \\
\phi(k+1)=A_{\phi d} \phi(k)+B_{\phi d} x(k)
\end{array}\right.
$$

The corresponding parameters of state space in Eqs (6) and (7) are derived by Matlab function pade. For simplicity, a state feedback is supposed instead of a state observer in this study, since we are not concerned about an observer. Thus, $y(k)=I x(k)=x(k)$ where $I$ is identical matrix. Afterwards, mark $X(t)=x\left(t-t_{d}\right)$ likely.

Considering the packet dropout segments of both the control input and measured output, it is impossible to acknowledge whether packet dropout happens or not in control input. Besides, by checking the validity of the data transmitted, the occurrence of the packet dropout in measured output is possible to be detected. Therefore, two different measures are proposed for each packet dropout segments. Before that, the property of the dropout functions $f(t), g(t)$ should be learnt first. It is obvious that dropout functions $f(t), g(t)$ are stochastic uncertainties that are unable to learn, and according to that, it is rational to discretize $f(t), g(t)$ as $F(k), G(k)$, which are also binary stochastic functions.

\section{Remark 1}

On the control input's side, a compensator of zeroorder holder is proposed, and the data of control input transmitted would not drop to zero when packet dropout happens, but keep the same with the last time, that is,

$$
u_{d}(k)=F(k) u_{c}(k)+[1-F(k)] u_{d}(k-1)
$$

On the measured output's side, we propose a measure that the estimation updated time to time since the packet dropout happens or, if not, is detectable upon checking the data validity. If packet dropout happens, the measured output must be invalid. Therefore, an estimated measured output may be obtained by

$$
y_{c}(k) \approx \hat{X}(k)=\left.E[G(k)] X(k)\right|_{F(k-1)=1}+\left.\{1-E[G(k)]\} X(k)\right|_{F(k-1)=0}
$$

where $E[G(k)]=n / k_{k}, n$ is the times that $G(i)=1, i=1$, ..., $k$. Finally, we can get the augmented simultaneous functions (10) from Eqs (5)-(8) as

$$
\begin{aligned}
{\left[\begin{array}{c}
x(k+1) \\
u_{d}(k) \\
\phi(k+1) \\
\phi(k+1)
\end{array}\right] } & =\left[\begin{array}{cccc}
A_{d} & B_{d}[1-F(k)] D_{\phi} & B_{d} C_{\phi} & 0 \\
0 & 1-F(k) & 0 & 0 \\
0 & {[1-F(k)] B_{\phi d}} & A_{\phi d} & 0 \\
B_{\phi d} & 0 & 0 & A_{\phi d}
\end{array}\right] \\
& *\left[\begin{array}{c}
x(k) \\
u_{d}(k-1) \\
\phi(k) \\
\phi(k)
\end{array}\right]+\left[\begin{array}{c}
F(k) B_{d} D_{\phi} \\
F(k) \\
F(k) B_{\phi d} \\
0
\end{array}\right] u_{c}(k)
\end{aligned}
$$

For simplicity, we designate Eq. (10) as

$$
\xi(k+1)=A_{\xi d}(k) \xi(k)+B_{\xi d}(k) u_{c}(k)
$$

where

$$
\begin{aligned}
\xi(k)=\left[\begin{array}{c}
x(k) \\
u_{d}(k-1) \\
\phi(k) \\
\phi(k)
\end{array}\right], A_{\xi d}(k) & =\left[\begin{array}{cccc}
A_{d} & B_{d}[1-F(k)] D_{\phi} & B_{d} C_{\phi} & 0 \\
0 & 1-F(k) & 0 & 0 \\
0 & {[1-F(k)] B_{\phi d}} & A_{\phi d} & 0 \\
B_{\phi d} & 0 & 0 & A_{\phi d}
\end{array}\right], \\
B_{\xi d}(k) & =\left[\begin{array}{llll}
F(k) B_{d} D_{\phi} & F(k) & F(k) B_{\phi d} & 0
\end{array}\right]^{T}
\end{aligned}
$$

\section{Control strategy}

An enhanced model predictive controller is proposed since the reconfigured transition function (11) is timevariant and multi-parametric, and such a prediction model is not supported by a common model predictive controller. Therefore, third-party Matlab toolbox YALMIP [15], a general parser for linear matrix inequalities, and multiparametric programming solver MPT3 (Multi-Parametric Toolbox) are introduced [16]. Firstly, the control target may be written as

$$
\begin{aligned}
& \arg \min _{u_{c}(0)} \sum_{k=0}^{N-1}\left[\Delta x^{T}(k) Q \Delta x(k)+u_{c}^{T}(k) R u_{c}(k)\right] \\
& \quad+\Delta x^{T}(N) P \Delta x(N)
\end{aligned}
$$

subject to

$$
\begin{gathered}
\xi(k+1)=A_{\xi d}(k) \xi(k)+B_{\xi d}(k) u_{c}(k) \\
x(k) \in\left[x_{\text {min }}, x_{\text {max }}\right], u_{c}(k) \in\left[u_{\text {cmin }}, u_{c \max }\right], \\
F(k) \in\{0,1\}
\end{gathered}
$$

where $\Delta x=x-x_{\text {ref }}, x_{\text {ref }}$ is the reference signal; $Q, R$ and $\mathrm{P}$ are weighting constants; $x_{\min }, x_{\max }$ and $u_{c \min }, u_{c \max }$ are lower and upper limits of $x(k)$ and $u_{c}(k)$, respectively; and $N$ is the predictive horizon of the model predictive controller.

Notice the result of optimization (13a) is not lucid to be achieved, and an effective method to handle optimization function (13a) shown in [17] is rewriting it as

$\min \omega$

subject to 


$$
\begin{aligned}
& \sum_{k=0}^{N-1}\left[\Delta x^{T}(k) Q \Delta x(k)+u_{c}^{T}(k) R u_{c}(k)\right] \\
& \quad+\Delta x^{T}(N) P \Delta x(N) \\
& \quad<\omega
\end{aligned}
$$

where $\omega$ is an epigraph variable. Therefore, the optimization problem (13a) is reformed into a linear matrix inequality (LMI) as is shown in formula (14). There have been plenty of studies and tutorials about how to solve LMI problems. Unconstrained ones are usually solved by the Riccati equation [18], while constrained ones may be expanded into a high-order LMI via the Schur complement then solved by Matlab toolboxes [19].

However, on the occasion of NCS of this study, the prediction model (13b) is uncertain and stochastic and the optimization problem is impossible to be solved instinctively by a conventional method. In a view of robustness, all possible states of prediction model (13b) in future should be taken into consideration. Still more, the uncertainty of transition function (13b) at time $k$ may be regarded as a linear parametric-varying function according to parameter $F(k)$; hence,

$$
\begin{gathered}
\left.A_{\xi d}(k)\right|_{F(k)=1}=\left[\begin{array}{cccc}
A_{d} & 0 & B_{d} C_{\phi} & 0 \\
0 & 0 & 0 & 0 \\
0 & 0 & A_{\phi d} & 0 \\
B_{\phi d} & 0 & 0 & A_{\phi d}
\end{array}\right], \\
\left.A_{\xi d}(k)\right|_{F(k)=0}=\left[\begin{array}{cccc}
A_{d} & B_{d} D_{\phi} & B_{d} C_{\phi} & 0 \\
0 & I & 0 & 0 \\
0 & B_{\phi d} & A_{\phi d} & 0 \\
B_{\phi d} & 0 & 0 & A_{\phi d}
\end{array}\right], \\
\left.B_{\xi d}(k)\right|_{F(k)=1}=\left[\begin{array}{llll}
B_{d} D_{\phi} & I & B_{\phi d} & 0
\end{array}\right]^{T}, \\
\left.B_{\xi d}(k)\right|_{F(k)=0}=\left[\begin{array}{llll}
0 & 0 & 0 & 0
\end{array}\right]^{T}
\end{gathered}
$$

Furthermore, uncertain variables $\theta_{0}(k)$ and $\theta_{1}(k)$ are introduced as

$$
\begin{aligned}
& \left\{\begin{array}{l}
\theta_{0}(k)=0 \\
\theta_{1}(k)=1
\end{array}, F(k)=1\right. \\
& \left\{\begin{array}{l}
\theta_{0}(k)=1 \\
\theta_{1}(k)=0
\end{array}, F(k)=0\right.
\end{aligned}
$$

Therefore, transition function (13b) may be rewritten in linear parametric-varying (LPV) format [20] which is supported by YALMIP as follows

$$
\begin{aligned}
\xi(k+1) & =\left.\theta_{1}(k) A_{\xi d}(k)\right|_{F(k)=1} \xi(k)+\left.\theta_{0}(k) A_{\xi d}(k)\right|_{F(k)=0} \xi(k) \\
& +\left.\theta_{1}(k) B_{\xi d}(k)\right|_{F(k)=1} u_{c}(k)+\left.\theta_{0}(k) B_{\xi d}(k)\right|_{F(k)=0} u_{c}(k)
\end{aligned}
$$

Notice the order of LMIs may grow rapidly due to the predictive horizon and the LPV format, making the solving computation expensive and unreliable. In order to increase the solving speed and reduce the computational expense, we introduce a discrete dynamic programming method which solves programming problems optimally by breaking them into sub-problems and finding the optimal solution recursively [21].

For the programming problem of dynamics $x_{i+1}=f\left(x_{i}\right.$, $u_{i}$ ) with cost function,

$$
\begin{aligned}
J_{0}\left(x, U_{0}\right) & =\sum_{i=0}^{N-1} l\left(x_{i}, u_{i}\right)+l_{f}\left(x_{N}\right), \\
U_{0} & =\left\{u_{0}, u_{1}, \ldots, u_{N-1}\right\}
\end{aligned}
$$

where $l$ is the running cost and $l_{f}$ is the final cost. Define partial cost function as

$$
\begin{aligned}
J_{i}\left(x, U_{i}\right) & =\sum_{j=i}^{N-1} l\left(x_{j}, u_{j}\right)+l_{f}\left(x_{N}\right), \\
U_{i} & =\left\{u_{i}, u_{i+1}, \ldots, u_{N-1}\right\}
\end{aligned}
$$

Thus, the value function may be written as

$$
V(x, i)=\min _{U_{i}} J_{i}\left(x, U_{i}\right)
$$

when $i=0, V(x, 0)=\min J_{0}\left(x, U_{0}\right)$ is the final objective of programming problem. Notice the value function (20) may be decomposed into the Bellman equation format [22]. Therefore,

$$
\begin{aligned}
& V(x, i)=\min _{U_{i}}\left[l\left(x_{i}, u_{i}\right)+V(x, i+1)\right] \\
& =\min _{u_{i}}\left[l\left(x_{i}, u_{i}\right)+V\left(f\left(x_{i}, u_{i}\right), i+1\right)\right]
\end{aligned}
$$

Conclusively, in this study, running cost is $\Delta x^{T}(k) Q \Delta x(k)$ $+u_{c}^{T}(k) R u_{c}(k)$, and final cost is $\Delta x^{T}(k) P \Delta x(N)$; the programming problem of the model predictive controller may be solved by solving the partial value function $V(x, i)$ recursively from $i=N-1$ to $i=0$. The final result of the control input is $u_{0}$, which is obtained by solving the final objective $V(x, 0)$.

Notice the programming problems in (13), (16) and (17) are not supported by an official toolbox of Matlab, while third-party solvers such as MPT3, SeDuMi, etc. are useful extensions of Matlab in solving LMIs. The steps are elaborated as follow. First, the NCS is modelled and reconfigured into an LPV model, then the LPV model is decomposed into a dynamic programming of Matlab codes, and YALMIP parses the Matlab codes, enumerating all possible occasions and eliminating the uncertain variables $\theta_{0}(k)$ and $\theta_{1}(k)$. Finally, multi-parametric LMIs in the programme is handled and solved by MPT3.

\section{Results and discussions}

In order to demonstrate the performance of the proposed method, two simulations are presented. The first one is based on a fixed-wing aircraft model. In this simulation, step responses of attitude are studied in order to show feasibility and stability of the proposed method. A numeric simulation of the second order LTI 


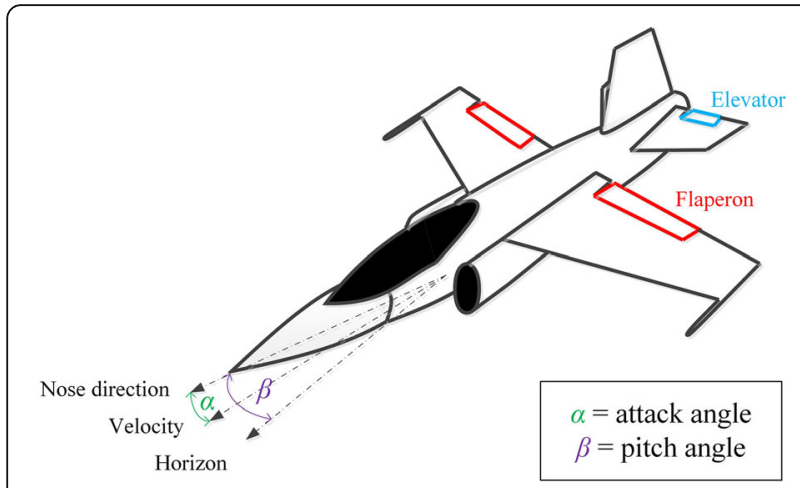

Fig. 3 Brief diagram of aircraft model of (22)

system is carried out in the other example, where the robustness as well as real-time property of the proposed method is studied.

Example 1

Consider a MIMO aircraft system as the target plant in NCS, with state space as [23]

$$
\left\{\begin{array}{c}
x(t)=\left[\begin{array}{cccc}
-0.0151 & -60.565 & 0 & -32.174 \\
-0.0001 & -1.3411 & 0.9929 & 0 \\
0.00018 & 43.254 & -0.8694 & 0 \\
0 & 0 & 1 & 0
\end{array}\right] \\
* x(t)+\left[\begin{array}{ccc}
-2.516 & -13.136 \\
-0.1689 & -0.2514 \\
-17.251 & -1.5766 \\
0 & 0
\end{array}\right] u(t) \\
y(t)=\left[\begin{array}{llll}
0 & 1 & 0 & 0 \\
0 & 0 & 0 & 1
\end{array}\right] x(t)
\end{array}\right.
$$

The aircraft model is shown in Fig. 3. Control inputs $u(t)$ are elevator and flaperon angles shown in Fig. 3, ranging between $\pm 25^{\circ}$ and $\pm 75^{\circ}$, respectively, while measured outputs $y(t)$ are attack and pitch angles ranging between $\pm 1^{\circ}$ and $\pm 75^{\circ}$, respectively, which are also designated as $\alpha$ and $\beta$ in Fig. 2. The sampling period also known as transmission interval in this study is $0.025 \mathrm{~s}$.

Step response results of NCS in Fig. 2 with target plant (22) are shown in Fig. 4, which is regulated by a conventional model predictive controller. Time delay is $0.1 \mathrm{~s}$
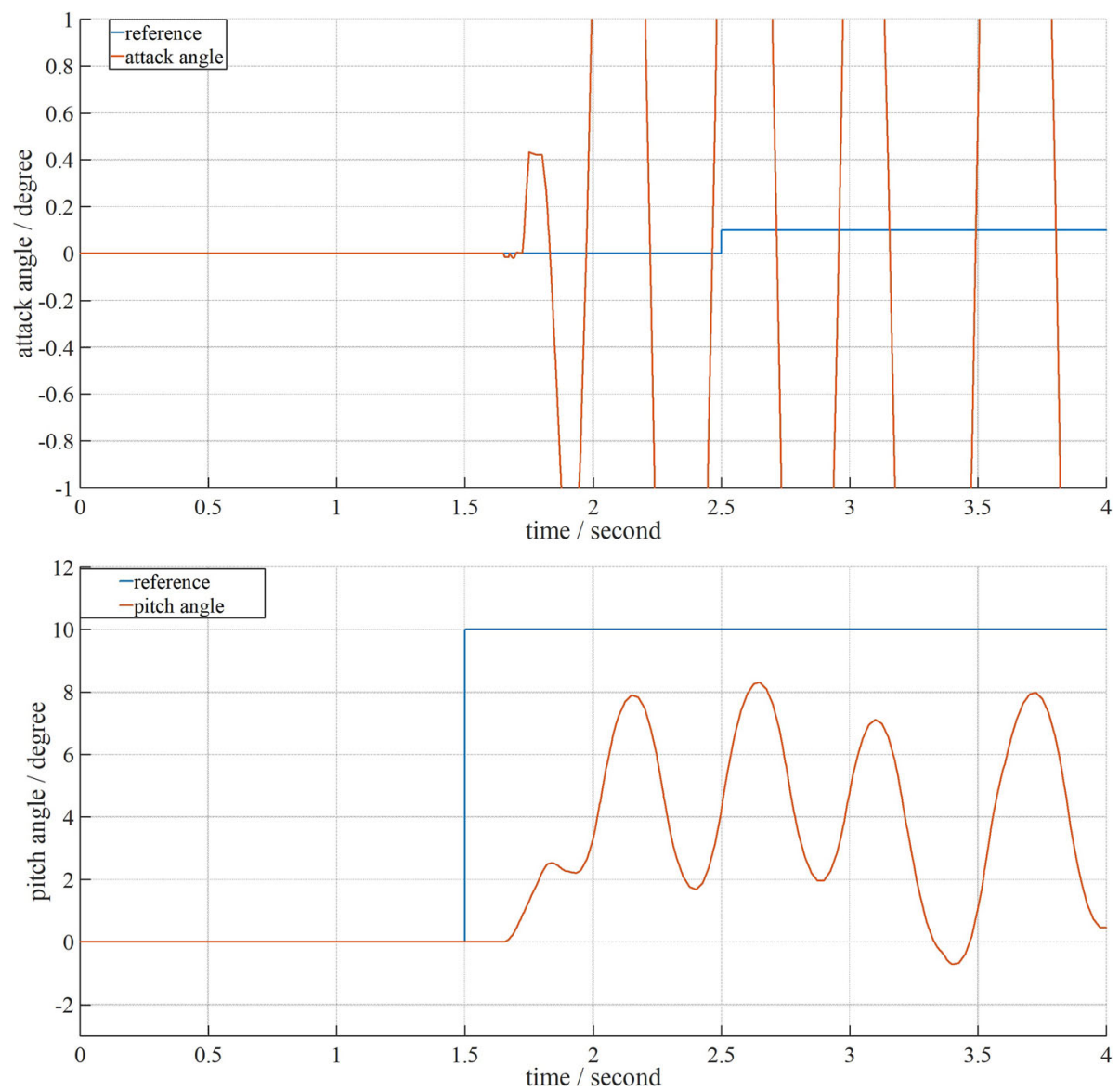

Fig. 4 Step responses of networked control target plant (22) by a conventional model predictive controller. a Waveform of attack angle. $\mathbf{b}$ Waveform of pitch angle 
and dropout rate is 0.1 of both control input and measured output networks.

The latency and stochasticity in NCS incur severe instability of studied flight system as is shown in Fig. 4. In order to demonstrate the advantages of the proposed method, simulation results employing a proposition under severer conditions are shown in Fig. 5, of which delayed time is $0.15 \mathrm{~s}$ and the dropout rate is 0.25 .

Variant prediction horizons are inspected as shown in Fig. 5, from 3 to 5 . It is obvious that all of the predictive horizons inspected can keep the NCS stable. As the prediction horizon increases, the undershoot of the attack angle around $1.7 \mathrm{~s}$ in Fig. 5a is smoothed gradually and the vibration of the pitch angle around $2.3 \mathrm{~s}$ in Fig. $5 \mathrm{~b}$ is flattened. The performance is adequately excellent when the prediction horizon grows as large as 5 .

Control inputs of the elevator and flaperon angle using the proposed method are shown in Fig. 6, of which the prediction horizon is fixed to 5 . The curves of control inputs after the packet dropout segment are well compensated by the proposed zero-order holder compensator as shown in Fig. 6a. The values may not drop out to zero when the packet dropout happens in the packet dropout segment of Fig. 2. Figure 6b is the stairstep graph of the dropout function, and pack dropout happens when the value is 0 , vice versa.

Figure 7 demonstrates the step response curves of the proposed method as delayed time changes slightly. The parameters and solution of the proposed method are intended to deal with networked control target plant (22) under a delayed time of $0.15 \mathrm{~s}$. Results in Fig. 7 show that the whole control system stays stable when delayed time shifts to $0.2 \mathrm{~s}$, while the performance is slightly downgraded as some peak undershoots takes place in the attack angle as shown in Fig. 7a.

Example 2

Another numeric simulation is presented, in which the target plant is in the second order. Therefore, threedimensional graphs of the value and control input function
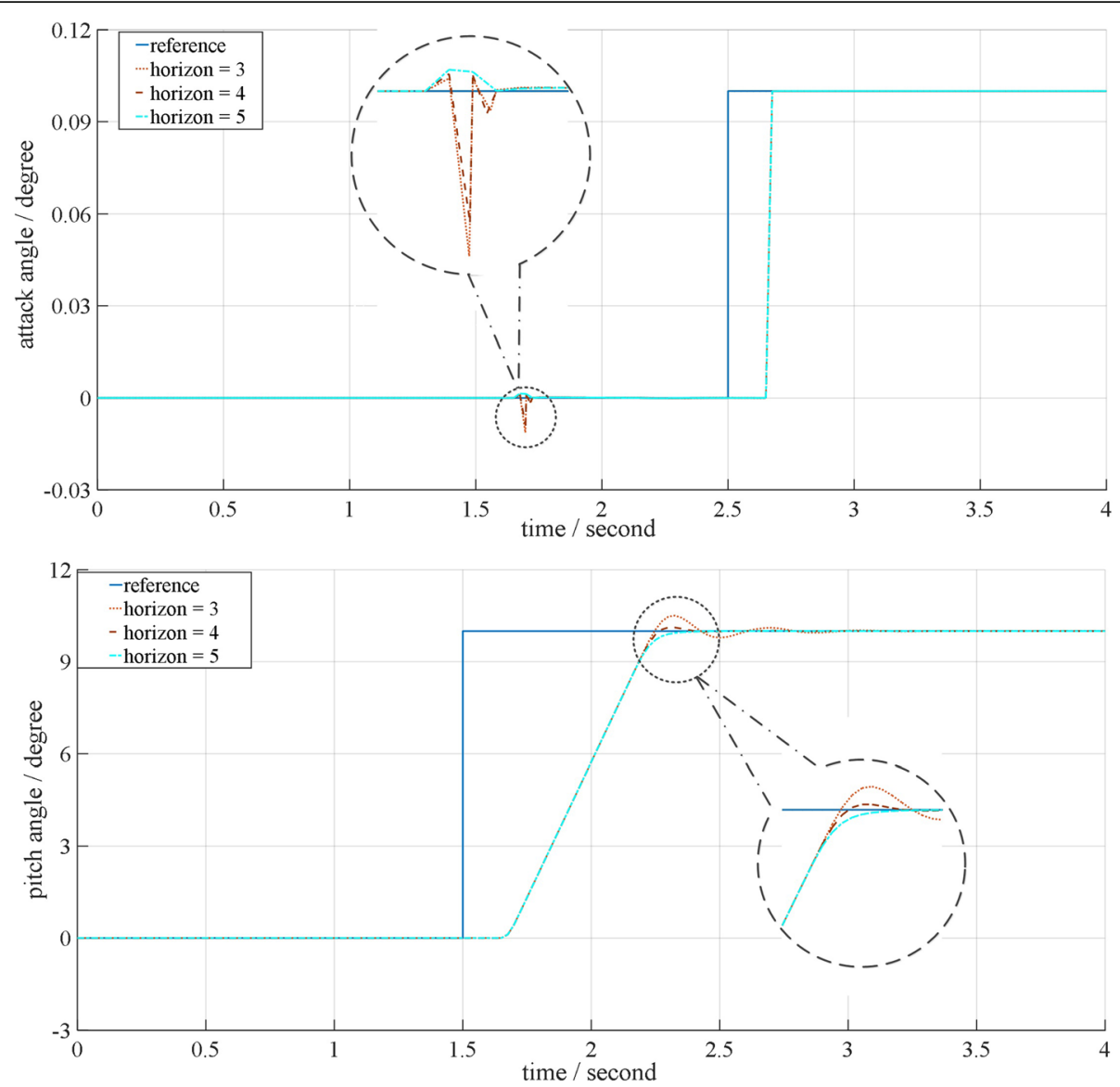

Fig. 5 Step responses of networked control target plant (22) by proposed method under variant prediction horizon from 3 to 5. a Partial waveforms of attack angle under variant prediction horizons. b Waveforms of pitch angle under variant prediction horizons 

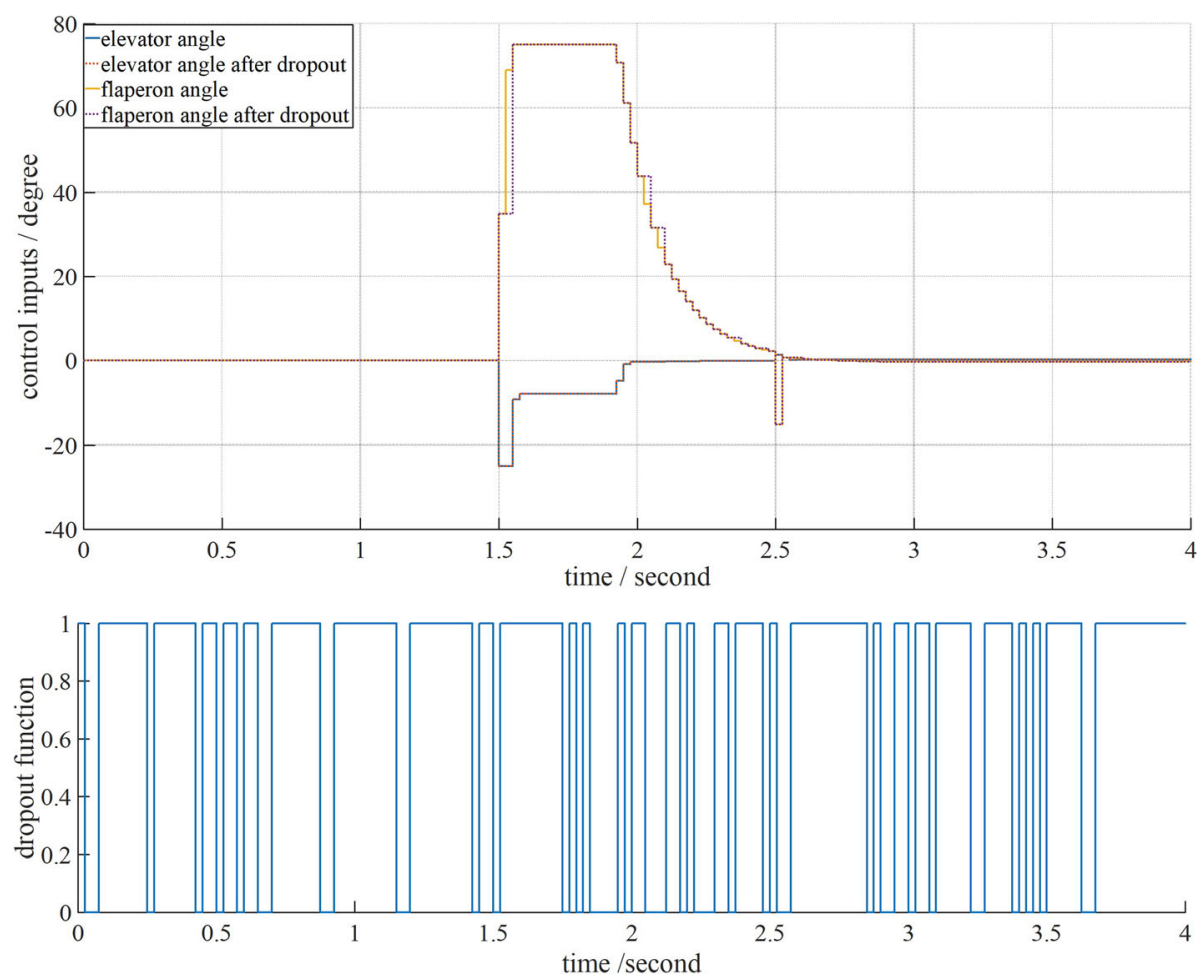

Fig. 6 a Control inputs of networked control target plant (22) by the proposed method under prediction horizon of 5, before and after the packet dropout segment; waveforms of control inputs. b Dropout function; values of dropout function

are possible to be plotted. In this way, more properties of the proposed method are possible to be acknowledged.

Consider the target plant of

$$
\left\{\begin{array}{l}
x(t)=\left[\begin{array}{cc}
2 & -1 \\
1 & 0
\end{array}\right] x(t)+\left[\begin{array}{l}
1 \\
0
\end{array}\right] u(t) \\
y(t)=\left[\begin{array}{ll}
1 & 0 \\
0 & 1
\end{array}\right] x(t) \\
z(t)=\left[\begin{array}{ll}
1 & 0 \\
0 & 0
\end{array}\right] x(t)
\end{array}\right.
$$

with transmission interval of $0.1 \mathrm{~s}$, control input and measured output delay both $0.3 \mathrm{~s}$, and prediction horizon $N$ of $4 . y(t)$ is the measured output and $z(t)$ is the desired output. Boundary constraints of states and control inputs are

$$
\left\{\begin{array}{l}
-5 \leq x(k) \leq 5 \\
-2 \leq u(k) \leq 2
\end{array}\right.
$$

The discretization and Pade approximation processes are carried out by Matlab functions. After the discretization and Pade approximation, we would like to solve the minimax problem of inequalities by zero initial states in order to derive the explicit solution, which is in the form of piecewise affine function sets in each critical region, including value function and control input function. The results are shown in Fig. 8.

The hyperplane in Fig. 8a demonstrates the cost function, which is employed to weigh the cost of a robust control strategy. When comparing to the simulation result of [24], the proposed method is $3.67 \%$ less, which means the method in this paper has a remarkable robustness relatively. The hyperplane in Fig. $8 \mathrm{~b}$ is the control input function. The value of control input may be derived from the hyperplane when parameters of reference and target plant are unchanged. Accordingly, the minimax problem is necessarily to be solved if only parameters of NCS are updated, while the conventional method needs to solve the programming problem every time. Moreover, a single minimax problem of a four-step model predictive control with a proposed discrete dynamic programming method takes $67.68 \mathrm{~s}$ by an AMD FX8310 CPU to solve, while it takes $211.24 \mathrm{~s}$ for the same control strategy without a discrete dynamic programming method. As a result, the proposed method can effectively save more time of solving; however, the algorithm needs further optimized to make such NCS more practical. A step response of this example is also presented as below.

The curves of the output in Fig. 9 take five steps to converge with the reference after the transmission delay of three steps, that is, $0.3 \mathrm{~s}$, while the control group of 

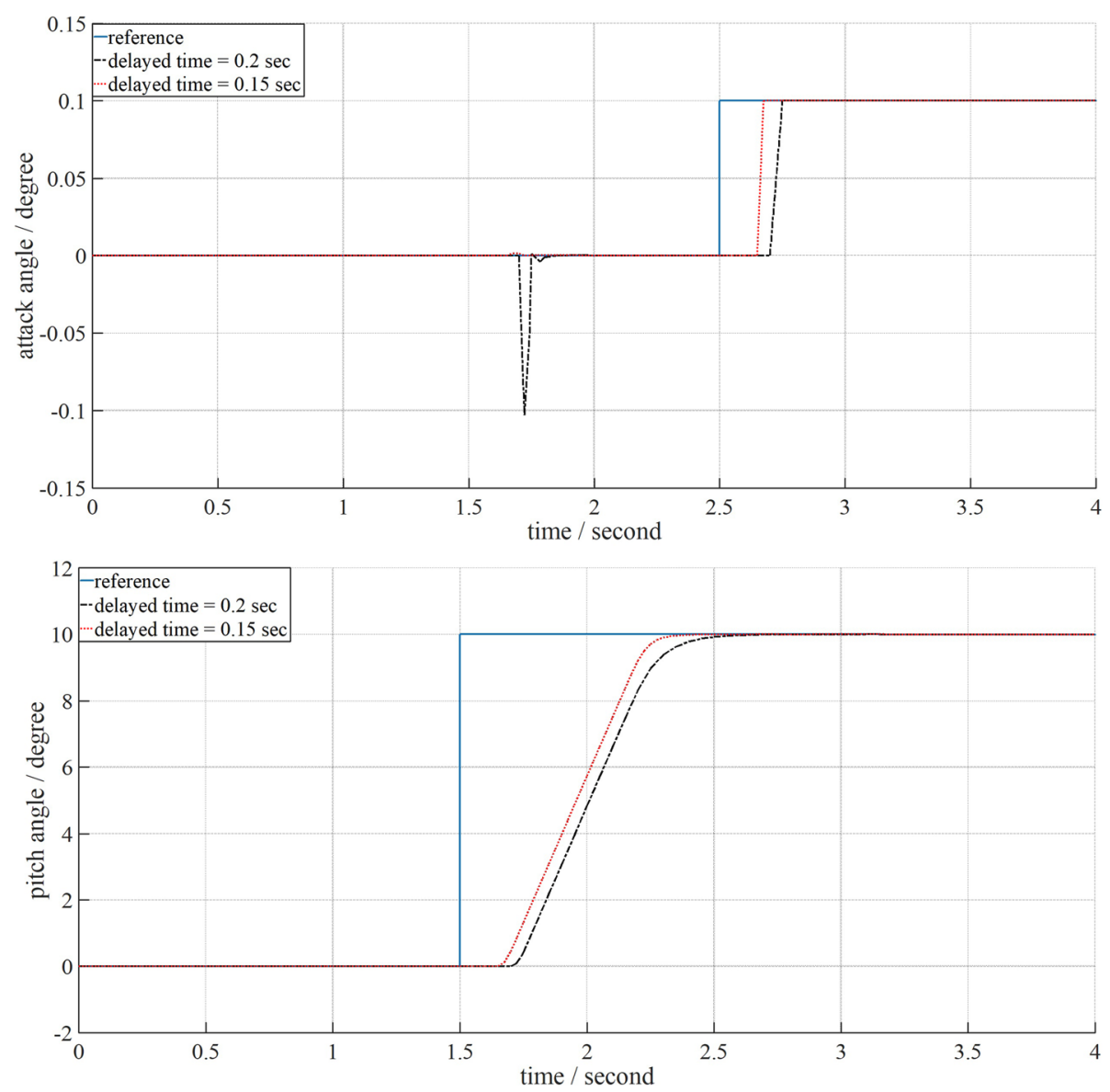

Fig. 7 Step responses of networked control target plant (22) under variant delayed times regulated by proposed method with prediction horizon of 5 and predictive model delay of $0.15 \mathrm{~s}$. a Waveforms of attack angle under variant delayed times. $\mathbf{b}$ Waveforms of pitch angle under variant delayed times

the PID control method in [24] underperforms unstable results in dealing with such NCSs, of which the curves diverge rapidly within several steps.

\section{Conclusion}

We have proposed a novel model predictive controller towards networked control systems featured with time delay and packet dropout in this paper. First of all, target plant and network are reconstructed and reformed into a reconfigured target plant, then in the modelling process, segments of time delay are eliminated by Pade approximation; moreover, a zeroorder holder-like compensator is utilized for packet dropout in control input, meanwhile packet dropout in the measured output is compensated by estimation. Followed the control target is reformed as a control problem of an uncertain time-varying multiparametric state space. Furthermore, in order to apply toolbox YALMIP and solver MPT3, such state space is rewritten as a linear parametric-varying prediction model. At last, a robust model predictive controller is completed by solving a constrained minimax problem of predictive cost function. The simulation results have shown a robust performance of the proposed method towards packet dropout and time delay in networked control system. The proposition in this study performs outstanding stability and also takes advantages in shortening solving time and enhancing the robustness of networked control systems with time delay and packet dropout. Meanwhile, we have considered an LTI target plant in this study for simplicity since the subject we focus on is NCSs with time delay and packet dropout. That does not mean the proposed method is not suitable for another target plant, say, nonlinear ones. Theoretically, the proposed method may be adapted by any target plant with progressive dynamics, which will be discussed in future studies. 

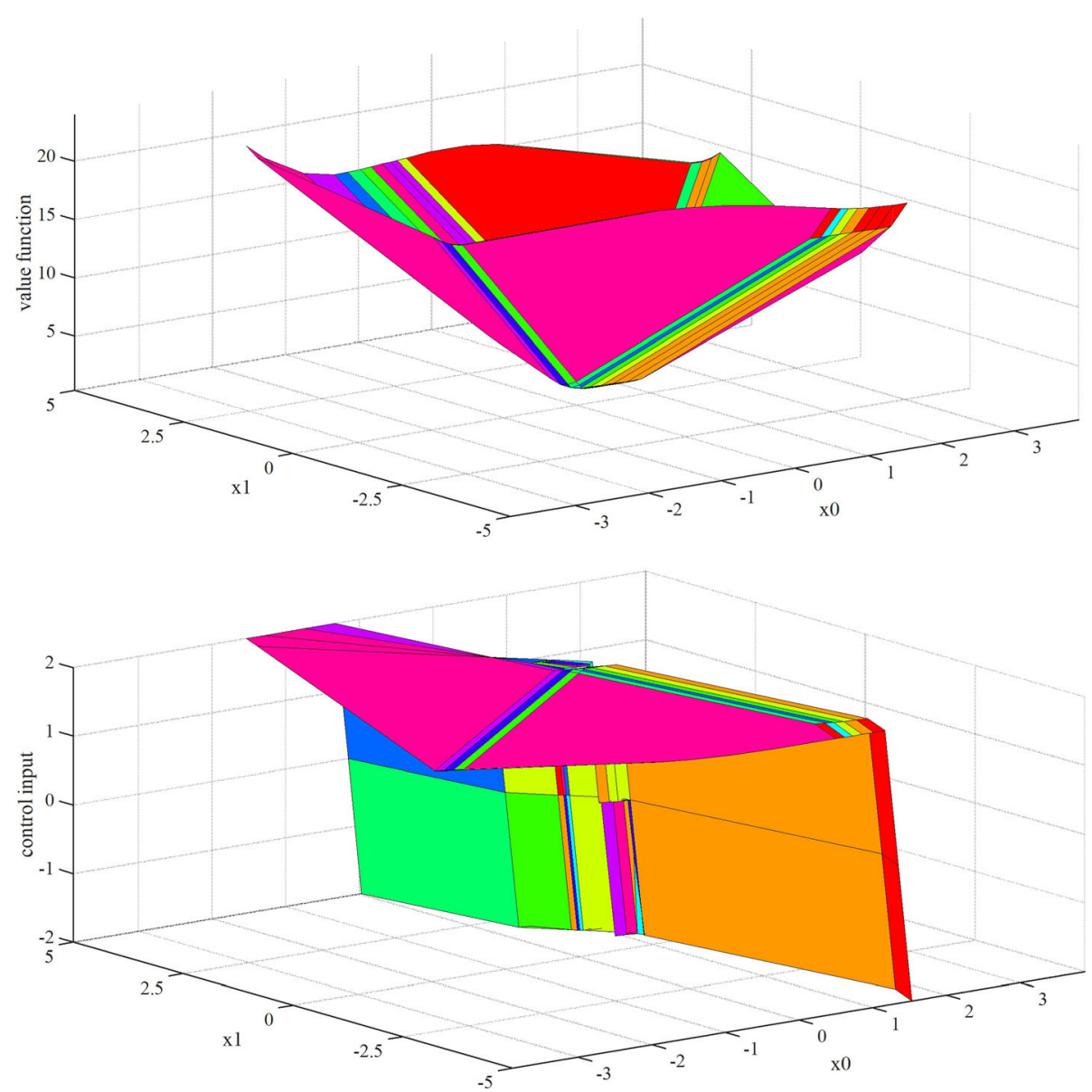

Fig. 8 Explicit solution of proposed method with regard to target plant (23). a Value function. b Control input function

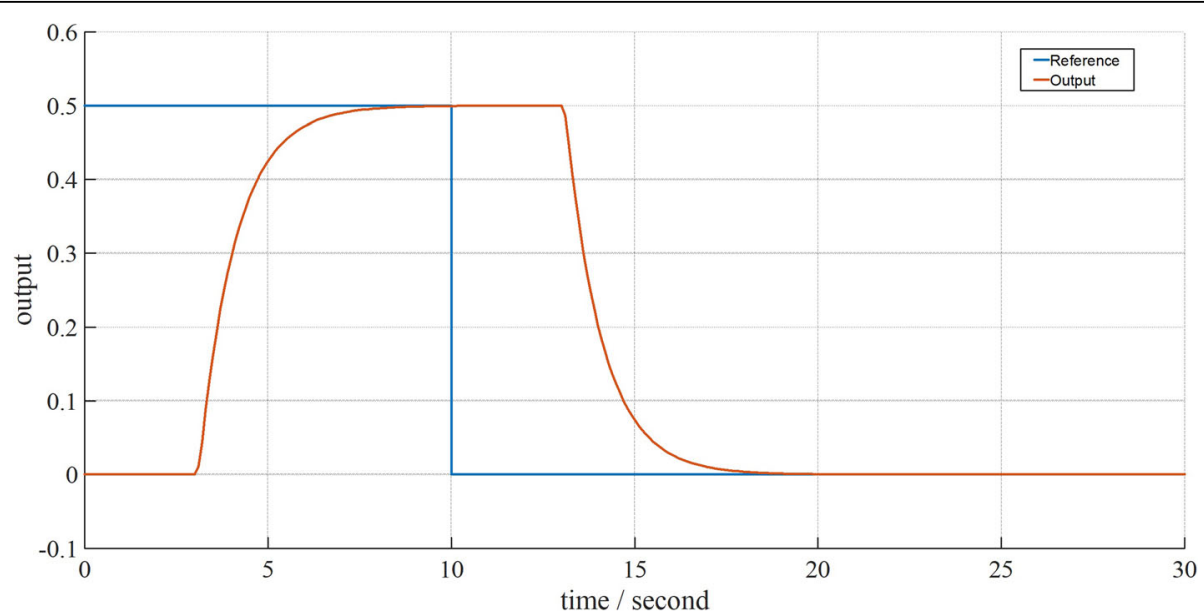

Fig. 9 Step response of target plant (23) using proposed method 


\section{Abbreviations}

LMI: Linear matrix inequality; LTI: Linear time-invariant; MIMO: Multi-input multi-output; MPT3: Multi-Parametric Toolbox; NCS: Networked control system; PID: Proportion integral differential; YALMIP: Yet Another Linear Matrix Inequalities Parser

\section{Acknowledgements}

The authors thank the editor and anonymous reviewers for their helpful comments and valuable suggestions.

\section{Authors' contributions}

All authors take part in the discussion of the work described in this paper These authors contributed equally to this work and should be considered co-first authors. All authors read and approved the final manuscript.

\section{Funding}

This work was supported by Ministry-level pre-research fund (No.6140004020105)

\section{Availability of data and materials}

The datasets used and/or analysed during the current study are available from the corresponding author on reasonable request.

\section{Competing interests}

The authors declare that they have no competing interest.

Received: 29 June 2019 Accepted: 11 September 2019

Published online: 14 February 2020

\section{References}

1. H. Chaouchi, The Internet of Things (Wiley-ISTE, London, 2010)

2. R. Aburukba, A.R. Al-Ali, N. Kandil, D. AbuDamis, in International Conference on Industrial Informatics and Computer Systems (CIICS 16). Configurable ZigBee-based control system for people with multiple disabilities in smart homes (2016), pp. 1-5. https://doi.org/10.1109//CCSII.2016.7462435

3. R.A. Gupta, M. Chow, Networked control system: Overview and research trends. IEEE Trans. On Industrial Electronics 57(7), 2527-2535 (2017). https:// doi.org/10.1109/TIE.2009.2035462

4. H. Mehrivash, M.H. Shafiei, in 2017 5th International Conference on Control, Instrumentation, and Automation (ICCIA). Robust model predictive control of discrete-time delayed positive systems (2017), pp. 150-154. https://doi.org/ 10.1109/ICCIAutom.2017.8258669

5. G. Torres, M. Velasco, P. Marti, J.M. Fuertes, E.X. Martin, in 39th Annual Conference of the IEEE industrial Electronics Society. An alternative discrete-time model for networked control systems with time delay less than the sampling period (2013), pp. 5627-5631. https://doi.org/10.1109/IECON.2013.6700056

6. J. Wu, T. Chen, Design of networked control systems with packet dropouts. IEEE Trans. on Automatic Control 52, 1314-1319 (2007). https://doi.org/10. 1109/TAC.2007.900839

7. P. Seiler, R. Sengupta, An Ho approach to networked control. IEEE Trans. on Automatic Control 50, 356-364 (2005). https://doi.org/10.1109/TAC.2005.844177

8. J. Wu, L. Zhang, T. Chen, in Proc. of the 26th Chinese Control Conference (CCC 07). An MPC approach to networked control design (2007), pp. 10-14. https://doi.org/10.1109/CHICC.2006.4346915

9. D. Nesic, A. Teel, Input-Output stability properties of networked control systems. IEEE Trans. on Automatic Control 49, 1650-1667 (2004). https://doi. org/10.1109/TAC.2004.835360

10. F. Yang, Q. Han, $\mathrm{H}_{\infty}$ control for networked systems with multiple packet dropouts. Information Sciences 252, 106-117 (2013). https://doi.org/10. 1016/J.INS.2013.06.043

11. W. Zhang, J. Bae, M. Tomizuka, Modified preview control for a wireless tracking control system with packet loss. IEEE/ASME Trans. On Mechatronics 20(1), 299-307 (Feb. 2015). https://doi.org/10.1109/TMECH.2013.2297151

12. J. Rawlings, D. Mayne, Model predictive control: theory and design (Nob Hill, Madison, 2009)

13. M.B.G. Cloosterman, L. Hetel, N. van de Wouw, W.P.M.H. Heemels, J. Daafouz, H. Nijmeijer, Controller synthesis for networked control systems. Automatica 46(10), 1584-1594 (2010)

14. R. Singh, V.M. Mishra, J. singh, in 2018 International Conference on Power Energy, Environment and Intelligent Control (PEEIC). Model order reduction via routh Hurwitz array and improved Pade approximations (2018), pp. 755758. https://doi.org/10.1109/PEEIC.2018.8665590

15. J. Lofberg, Minimax approaches to robust model predictive control (Linkoping Studies in Science and Technology. Dissertations, Linkoping, 2003), pp. 39-62

16. M. Herceg, M. Kvasnica, C.N. Jones, M. Morari, in 2013 European Control Conference (ECC). Multi-Parametric Toolbox 3.0 (2013), pp. 502-510. https:// doi.org/10.23919/ECC.2013.6669862

17. C.D. Aliprantis, K.C. Border, Infinite dimensional analysis: A hitchhiker's guide, 3rd edn. (Springer Science \& Business Media), pp. 8-10

18. D. Simon, J. Lofberg, T. Glad, Reference tracking MPC using dynamic terminal set transformation. IEEE Trans. On Automatic Control 59(10), 27902795 (2014). https://doi.org/10.1109/TAC.2014.2313767

19. Y. Lan, H. Huang, Observer-based robust control of time-delay uncertain systems with application to engine idle speed control. International Jounal of Dynamical Systems and Differential Equations 4(3), 274-285 (2012). https://doi.org/10.1504/IJDSDE.2012.049905

20. T. Besselmann, J. Lofberg, M. Morari, Explicit MPC for LPV systems: Stability and optimality. IEEE Trans. on Automatic Control 57(9), 2322-2332 (2012). https://doi.org/10.1109/TAC.2012.2187400

21. F. Borrelli, A. Bemporad, M. Morari, Predictive control for linear and hybrid systems (Cambridge University Press, London, 2015), pp. 181-196

22. J. Miao, Economic dynamics in discrete time (MIT Press, 2014), pp. 134-143

23. A. Bemporad, A. Casavola, E. Mosca, Nonlinear control of constrained linear systems via predictive reference management. IEEE Trans. on Automatic Control 42(3), 340-349 (1997). https://doi.org/10.1109/9.557577

24. Z. Wang, S. Fukushima, T. Hachino, in 20185 th International Conference on Information Science and Control Engineering (ICISCE). Model predictive control approach on packet dropout prevention of networked control systems (2018), pp. 839-843. https://doi.org/10.1109/ICISCE.2018.00176

\section{Publisher's Note}

Springer Nature remains neutral with regard to jurisdictional claims in published maps and institutional affiliations.

\section{Submit your manuscript to a SpringerOpen ${ }^{\circ}$ journal and benefit from:}

- Convenient online submission

- Rigorous peer review

- Open access: articles freely available online

High visibility within the field

- Retaining the copyright to your article

Submit your next manuscript at $\boldsymbol{\nabla}$ springeropen.com 